\title{
Factores de riesgo cardiovascular en pacientes intervenidos de estenosis carotídea en el Hospital 12 de Octubre
}

\author{
Raquel Manuel Garijo ${ }^{1}$, Mari Cruz Mora Pérez ${ }^{1}$, Cristina Brizuela Domínguez ${ }^{1}$, Raquel Bustamante Guerrero ${ }^{1}$. \\ ${ }^{1}$ Enfermera. Servicio de Angiología y Cirugía Vascular, Hospital Universitario 12 de Octubre. Madrid. España.
}

\author{
Correspondencia \\ Raquel Manuel Garijo \\ Correo electrónico: rqel@yahoo.es \\ Recibido: 07/05/2019 \\ Aceptado: 05/06/2019
}

\section{RESUMEN}

Objetivos. Analizar los factores de riesgo asociados a la estenosis carotídea en los enfermos operados por esta patología desde enero de 2013 hasta diciembre de 2017 en el Servicio de Cirugía Vascular del Hospital Universitario 12 de Octubre de Madrid.

Material y método. Estudio descriptivo, retrospectivo observacional, en el que se analizan las historias clínicas de los pacientes intervenidos de estenosis carotídea para identificar los factores de riesgo asociados a esta patología.

Resultados. 161 pacientes fueron intervenidos, con una media de edad de 72 años $\pm 9,68$. Un $80 \%$ fueron hombres ( $n=129)$ y un $20 \%$ mujeres ( $n=32$ ). El $44.7 \%$ de los casos tenía entre 70 y 80 años. En este rango se sitúa también el mayor porcentaje de enfermos sintomáticos, correspondiendo estos a un $55.3 \%(n=89)$ del total de la muestra. La hipertensión y la dislipemia fueron los factores más prevalentes. La sintomatología previa y la hipertensión guardan una relación significativa $(p \leq 0,05)$, así como el ser fumador o exfumador y ser hombre.

Discusión-conclusión. Los factores de riesgo identificados en la bibliografía fueron encontrados en la muestra de estudio. Todos presentaban algún factor de riesgo. El control de dichos factores es un aspecto importante del tratamiento médico.

\section{PALABRAS CLAVE}

Estenosis carotídea; Factores de riesgo.

\section{SUMMARY}

Background. The studies relate carotid stenosis with certain risk factors such as arterial hypertension, diabetes mellitus, dyslipidemia and smoking.

Goals. To analyze the risk factors associated with this pathology in the patients who were operated from January 2013 to December 2017 at the Vascular Surgery Unit of the University Hospital 12 de Octubre.

Methods. A descriptive, retrospective, observational study in which the clinical histories of patients undergoing carotid stenosis are analyzed to identify the risk factors associated with this pathology.

Results. 161 patients were operated, with an average age of 72 years $\pm 9.68 .80 \%$ were men $(n=129)$ and $20 \%$ were women ( $n=32$ ). In $44.7 \%$ of the cases, it was between 70 and 80 years old. In this age range is also the highest percentage of symptomatic patients, corresponding to $55.3 \%(n=89)$ of the total sample. Hypertension and dyslipidemia were the most prevalent factors. The previous symptomatology and hypertension have a significant relationship $(p \leq 0,05)$, as well as being a smoker or ex-smoker and being a man.

\section{KEYWORDS}

Carotid stenosis; Risk factors.

\section{Introducción}

La estenosis carotídea (ECA) es un importante factor de riesgo de accidente cerebrovascular (ACV) isquémico, ocasionando entre un 10 y un $15 \%$ de los ACV isquémicos (1).

Puede clasificarse en sintomática o asintomática en base a la presencia o ausencia de signos y síntomas cerebrovasculares. La gravedad de los síntomas es variable. Se puede presentar como ataque isquémico transitorio
(AIT) (por ejemplo, con pérdida visual monoocular brusca, típicamente "en cortina"), o bien presentarse como ACV constituido (2). No obstante, la gran mayoría de los pacientes con ECA, incluso si esta es severa, permanecen asintomáticos durante años e incluso indefinidamente. Solo se ha documentado una mayor incidencia de ictus cuando la estenosis muestra progresión y alta vulnerabilidad de la placa o cuando hay síntomas clínicos (3). 
Los objetivos de la prevención son limitar la progresión de la aterosclerosis y reducir el riesgo de ACV y otros sucesos vasculares (4). De este modo, las indicaciones para la revascularización dependen sobre todo del grado de la estenosis y de si el paciente ha experimentado recientemente síntomas neurológicos (5). La Figura 1 muestra una imagen radiológica de un paciente afecto de estenosis carotídea.

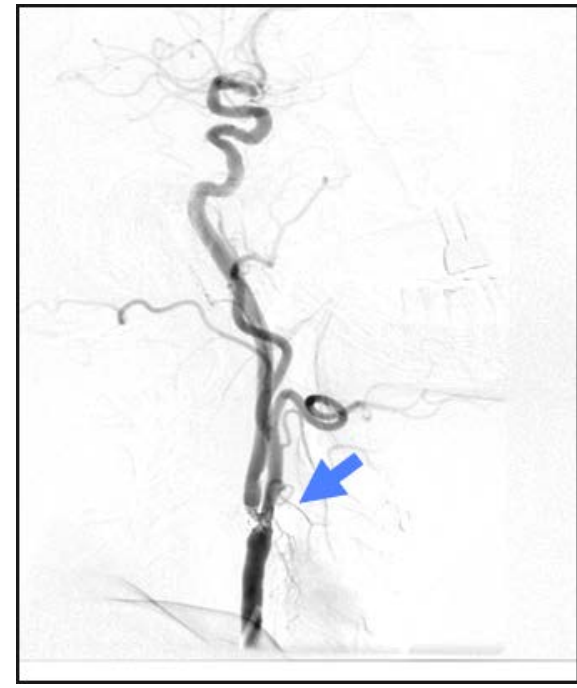

Fig. 1: Imagen radiológica de estenosis carotídea.

En la actualidad, las opciones para el tratamiento activo de la ECA son la endarterectomía carotídea quirúrgica y la terapia endovascular mediante angioplastia y stent carotídeo. No obstante, la revascularización de la carótida es únicamente un aspecto del tratamiento administrado a los pacientes con arteriopatía carotídea, ya que también es necesario un control de los factores de riesgo cardiovascular.

Son muchos los estudios que relacionan la estenosis carotidea con determinados factores de riesgo como la hipertensión arterial, la diabetes, la dislipemia y el hábito de fumar (6-11). Desde hace años se conoce la relación entre la presión arterial y el riesgo de desarrollo de ECA, de manera que por cada aumento de la presión arterial sistólica en $20 \mathrm{~mm}$ de $\mathrm{Hg}$ se duplica el riesgo de desarrollar esta patología (6). Asimismo, se sabe que el tabaco incrementa el riesgo de ictus isquémico, y este riesgo es proporcional a la duración e intensidad del tabaquismo (7).

La combinación de factores de riesgo aumenta adicionalmente la prevalencia de ECA (8) (9) y dicha patología se ha visto más frecuentemente en pacientes con enfermedad periférica vascular(10) (11) y enfermedad coronaria (12) (13).

El control de los factores de riesgo es, por tanto, esencial en la prevención primaria y secundaria del $\mathrm{ACV}$. Este control incluye: detección o diagnóstico de los factores de riesgo; abandono de hábitos nocivos como tabaquismo, consumo excesivo de alcohol, se- dentarismo, drogas, etc.; dieta (específica para cada perfil de riesgo) y control de la obesidad; antiagregantes plaquetarios; antihipertensivos (solo para hipertensos) y estatinas (para dislipémicos y no dislipémicos) (1)(14).

La optimización de la calidad del tratamiento médico en las últimas décadas ha llevado a una reducción del riesgo de ACV (15).

El objetivo de este estudio fue analizar los factores de riesgo asociados a la estenosis carotidea en los enfermos intervenidos por esta patología en el Servicio de Angiología y Cirugía Vascular del Hospital Universitario 12 de Octubre de Madrid.

\section{Material y métodos}

Estudio descriptivo, retrospectivo observacional, de enero de 2013 a diciembre de 2017, en el que se analizan las historias clínicas de los pacientes intervenidos de estenosis carotidea en el Servicio de Angiología y Cirugía Vascular del Hospital Universitario 12 de Octubre de Madrid. La recogida de datos la llevó a cabo el personal de enfermería perteneciente a la unidad de hospitalización de dicho servicio.

Se realizó una revisión de la bibliografía para determinar qué factores de riesgo se asocian con esta patología. De este modo, se establecieron como variables demográficas la edad y el sexo y, como variables clínicas, la hipertensión arterial, la diabetes mellitus, la hiperlipemia y el tabaquismo (fumadores y exfumadores). También se analizó si los pacientes habían estado sintomáticos o asintomáticos, considerando sintomáticos aquellos que habían tenido episodios de AIT o ACV en los seis meses previos a la intervención y que habían sido diagnosticados por el neurólogo especialista. Aquellos pacientes intervenidos de ambas carótidas se analizaron como paciente único.

Los resultados se expresan mediante frecuencia absoluta y relativa para las variables cualitativas, y media y desviación estándar para las variables cuantitativas. Se utilizó la prueba no paramétrica de $U$ de MannWhitney y el test de Chi-cuadrado para comparar si existían diferencias entre sexos con respecto a la presencia de factores de riesgo. Se proporcionará el estimador de la diferencia e intervalo de confianza al 95\%. Se consideraron estadísticamente significativos los valores de $p \leq 0,05$.

El modelo de predicción se realizó mediante regresión logística binaria, introduciendo como variable dependiente sintomático y como variables independientes los factores de riesgo. En aquellos casos en los que se vio una relación significativa se presenta el Odds Ratio (intervalo de confianza [IC] del 95\%).

Se utilizó el paquete estadístico SPSS versión 15.0 (SPSS Science, Chicago, Illinois, USA).

Se siguieron los principios éticos de la Declaración de Helsinki (World Medical Association. Declaration of Helsinki: ethical principles for medical research invol- 
ving human subjets, 2012), así como la normativa sobre acceso a Documentación e Información Clínica: Ley Orgánica 15/1999 sobre Protección de Datos de Carácter Personal y Ley 41/2002 reguladora de la Autonomía del paciente y de derechos y obligaciones en materia de información y documentación clínica.

\section{Resultados}

Durante nuestro periodo de estudio de enero de 2013 a diciembre de 2017 un total de 161 pacientes fueron intervenidos de ECA. La Figura 2 presenta la distribución de la muestra por sexo.

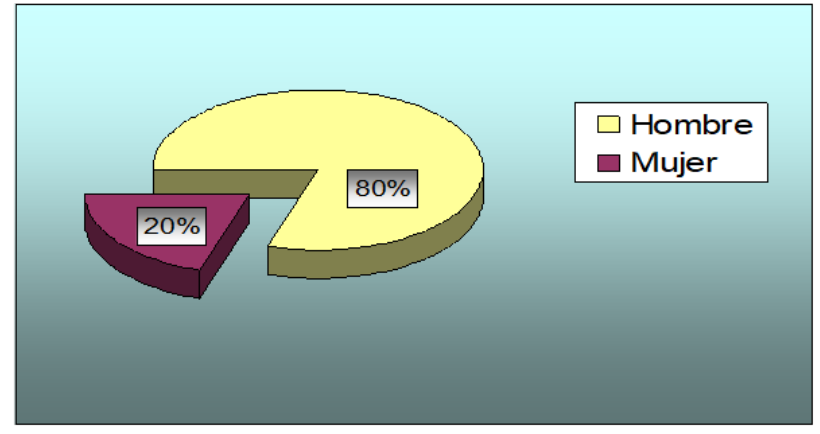

Fig. 2: Distribución de la muestra por sexo.

La media de edad \pm la desviación estándar (DE) fue de 72 años $\pm 9,68$ DE (rango de edad 43-90). En la Tabla 1 se muestran las edades agrupadas por rangos de edad y distribuidas por sexo. El mayor porcentaje de enfermos intervenidos, en ambos sexos, estaba en el rango entre los 70 y los 80 años, con un $44.72 \%$ de los casos $(n=72)$. Además, en este intervalo se situaba también el mayor porcentaje de enfermos sintomáticos: $39.33 \%(n=35)$.

Tabla 1. Distribución muestral por rangos de edad y sexo

\begin{tabular}{llcc}
\hline Sexo & Grupo de edad & Frecuencia (n) & (\%) \\
\hline \multirow{3}{*}{ Hombres } & Menos de 50 & 4 & 2.48 \\
\cline { 2 - 4 } & $(50-60)$ & 19 & 11.80 \\
\cline { 2 - 4 } & $(60-70)$ & 25 & 15.53 \\
\cline { 2 - 4 } & $(70-80)$ & 58 & 36.02 \\
\cline { 2 - 4 } & Más de 80 & 23 & 14.29 \\
\hline \multirow{3}{*}{ Mujeres } & Menos de 50 & 1 & 0.62 \\
\cline { 2 - 4 } & $(50-60)$ & 2 & 1.24 \\
\cline { 2 - 4 } & $(60-70)$ & 8 & 4.97 \\
\cline { 2 - 4 } & $(70-80)$ & 14 & 8.70 \\
\cline { 2 - 4 } & Más de 80 & 7 & 4.35 \\
\hline Total & & 161 & 100 \\
\hline
\end{tabular}

En cuanto a la presencia de factores de riesgo cardiovascular, el $36.02 \%$ de los pacientes fueron diabéti$\cos (n=8)$, el $76.40 \%$ hipertensos $(n=123)$, el $69.57 \%$ dislipémicos, $(n=112)$, un $26.09 \%(n=42)$ exfumadores y un $31.06 \%(n=50)$ fumadores. La Tabla 2 muestra la distribución de los factores de riesgo por sexo, donde se observa que la hipertensión y la dislipemia eran los factores de riesgo más frecuentes.

Con relación a la aparición de sintomatología previa, 89 pacientes habían desarrollado síntomas (55\%). En este grupo, como en la muestra total, los factores de riesgo más prevalentes eran también la hipertensión arterial y la diabetes (Tabla 3). Se vio además una asociación entre la aparición de sintomatología previa y ser hipertenso ( $p=0.0268$ ) (OR 2.45 [IC95\% 1.12-5.39]).

Comparando los factores de riesgo por sexo, solo había una relación significativa entre fumar y haber fumado y ser hombre $(p=0.0013)$.

\section{Discusión}

Estos resultados muestran que la prevalencia de estenosis carotídea aumenta con la edad, lo que está en concordancia con otros trabajos publicados (16). Los datos analizados indican que el grueso de los pacientes operados se sitúa en el rango entre 70 y 80 años, encontrándose también en este intervalo, el mayor porcentaje de pacientes sintomáticos. De Weerd et al. (17) identifica que la estenosis carotídea severa, definida por un $70 \%$ o más, está presente en un $0.1 \%$ de los hombres menores de 50 años y en un 3.1\% de los hombres mayores de 80 años. En ese mismo estudio, el $0 \%$ de las mujeres menores de 50 años, y el $0.9 \%$ de las mujeres mayores de 80 años presentaban estenosis carotídea severa. De la misma manera, los hombres padecen con mayor frecuencia esta enfermedad, probablemente debido a una mayor exposición a los factores de riesgo.

En nuestro estudio la hipertensión arterial y la dislipemia son los factores de riesgo con mayor prevalencia, tanto en pacientes sintomáticos como en asintomáticos. Estos datos coinciden con el estudio de Chiquete et al. (18), que indica que, después de la edad $\geq 55$ años, el factor de riesgo más prevalente es la hipertensión, seguida de la dislipemia, la obesidad, la diabetes y el tabaquismo. Otros estudios señalan que una exposición a dichos factores durante 15años o más influye fuertemente en la aparición de ECA (9).

Además, el estudio comparativo entre haber desarrollado síntomas antes de la intervención y presentar algún factor de riesgo, mostró una asociación significativa con la hipertensión arterial. Esto está en concordancia con otros trabajos, en los que se indica que la presión arterial alta incrementa el riesgo de ACV y que una reducción de la misma disminuye dicho riesgo (19)(20). Igualmente, la hipertensión es el principal factor de riesgo de la enfermedad vascular y su prevalencia aumenta con la edad (2).

En cuanto a la dislipemia, es un factor de riesgo creciente para el desarrollo de enfermedad ateromatosa. Los datos del estudio LIPID sobre el tratamiento con estatinas muestran una reducción del riesgo de ACV de entre un $25 \%$ y el $32 \%$ en los pacientes tratados (24). 


\begin{tabular}{|c|c|c|c|c|c|}
\hline \multirow[t]{2}{*}{ Comorbilidades asociadas } & \multicolumn{2}{|c|}{ Hombres } & \multicolumn{2}{|c|}{ Mujeres } & \multirow[t]{2}{*}{$\mathbf{p}$} \\
\hline & $\mathrm{n}$ & (\%) & $\mathrm{n}$ & (\%) & \\
\hline Diabetes mellitus & 44 & 34,11 & 14 & 43,75 & 0.3126 \\
\hline Hipertensión arterial & 98 & 75.97 & 25 & 78.13 & 1.0000 \\
\hline Dislipemia & 87 & 67.44 & 25 & 78.13 & 0.2877 \\
\hline Fumador actual & 44 & 34.11 & 6 & 18.75 & \\
\hline Exfumador & 38 & 29.46 & 4 & 12.50 & \\
\hline Fumador o exfumador & & & & & 0.0052 \\
\hline \multicolumn{6}{|c|}{ Tabla 3. Factores de riesgo en los enfermos sintomáticos $(n=89)$} \\
\hline Comorbilidades asociadas & & encia ( $n)$ & & $(\%)$ & p \\
\hline Diabetes mellitus & & 28 & & 31.46 & 0.1908 \\
\hline Hipertensión arterial & & 62 & & 69.66 & 0.0268 \\
\hline Dislipemia & & 60 & & 67.42 & 0.6058 \\
\hline Fumador actual & & 19 & & 21.35 & \\
\hline Exfumador & & 31 & & 34.83 & \\
\hline Fumador o exfumador & & 50 & & & 0.2625 \\
\hline
\end{tabular}

La diabetes mellitus es otro factor de riesgo importante para el desarrollo de enfermedad ateromatosa. En nuestro estudio, el número de enfermos con diabetes fue el factor de riesgo menos prevalente. Estos datos difieren del estudio de Angelis $M$ et al. (25), que señala que los sujetos con diabetes tipo 2 tienen tres veces más riesgo de desarrollar ECA asintomática que los no diabéticos, y que el riesgo relativo de desarrollar un ACV asociado con DM es de 1,4 a 1,7 (21).

En la muestra estudiada se ha visto que un número considerable de pacientes ( $n=92$ ) han consumido o consumen tabaco. El análisis comparativo entre factores de riesgo y sexo muestra una correlación significativa en el hábito de fumar y ser hombre. El estudio de Ruijun et al. (24), establece que el hábito de fumar es un factor de riesgo asociado a la formación de estenosis carotídea extracraneal, incrementándose el riesgo en 1,1\% por cada año de consumo de tabaco.

Debido a que la muestra analizada es pequeña, para poder extrapolar los datos, se debería llevar a cabo un estudio más amplio.

El análisis realizado y la bibliografía consultada muestran que este tipo de pacientes requieren una adecuada educación sanitaria que ayude a disminuir los factores de riesgo modificables asociados con esta patología. Enfermería, en todos los ámbitos y niveles asistenciales, debe dirigir sus intervenciones educativas hacia aspectos como el cese del hábito de fumar, evitar el sedentarismo, seguir una dieta adecuada y mantener una buena adherencia al tratamiento médico prescrito.

\section{Conclusiones}

Los resultados de este estudio están en concordancia con la literatura consultada relativa a la presencia de factores de riesgo cardiovascular entre pacientes intervenidos de estenosis carotídea. Todos los pacientes estudiados presentan algún factor de riesgo cardiovascular: diabetes, hipertensión arterial, tabaquismo y/o dislipemia.

\section{Conflicto de intereses}

Los autores declaran no presentar conflictos de intereses.

\section{Bibliografía}

1. Sposato LA, Klein F. Enfermedad carotídea aterosclerótica extracraneal. Neurol Argentina. 2011;3(1): 26-53.

2. Consenso de Estenosis Carotídea. Rev Argentina Cardiol. 2006;74(2):160-74.

3. Castilla-Guerra L, Fernández-Moreno MC, SerranoRodríguez L. Manejo actual de la estenosis carotídea asintomática. Rev Clínica Española. 2015; 215(4):224-9.

4. Mas JL. Revascularización de la arteria carótida interna. Rev Española Cardiol. 2007; 60 (8): 861-71.

5. Ricotta JJ, Aburahma A, Ascher E, Eskandari M, Faries P, Lal BK. Updated Society for Vascular Surgery guidelines for management of extracranial carotid disease: Executive summary. J Vasc Surg. 2011;54(3):832-6. 
6. Wilson PWF, Hoeg JM, D'Agostino RB, Silbershatz $H$, Belanger AM, Poehlmann $\mathrm{H}$, et al. Cumulative Effects of High Cholesterol Levels, High Blood Pressure, and Cigarette Smoking on Carotid Stenosis. N Engl J Med. 1997; 337(8):516-22.

7. Goldstein LB, Bushnell CD, Adams RJ, Appel LJ, Braun LT, Chaturvedi $S$, et al. Guidelines for the Primary Prevention of Stroke. Stroke. 2011; 42 (2): 517-84.

8. Signorelli SS, Di Pino L, Fichera G, Celotta G, Pennisi $G$, Marchese $G$, et al. Ultrasound diagnosis of carotid artery lesions in a population of asymptomatic subjects presenting atherosclerosis risk factors. J Stroke Cerebrovasc Dis. 2004 ;13(3):95-8.

9. Kaul S, Alladi S, Mridula KR, Bandaru VCSS, Umamashesh M, Anjanikumar D, et al. Prevalence and risk factors of asymptomatic carotid artery stenosis in Indian population: An 8-year follow-up study. Neurol India. 2017;65(2):279-85.

10. Norgren L, Hiatt WR, Dormandy JA, Nehler MR, Harris KA, Fowkes FG, et al. Inter-society consensus for the management of peripheral arterial disease. Vol. 26, International angiology: a journal of the International Union of Angiology. 2007. p. 81-157.

11. Bez LG, Navarro TP. Study of carotid disease in patients with peripheral artery disease. Rev Col Bras Cir. 41(5):311-8.

12. Ahmed B, Al-Khaffaf $\mathrm{H}$. Prevalence of Significant Asymptomatic Carotid Artery Disease in Patients with Peripheral Vascular Disease: A Meta-Analysis. Eur J Vasc Endovasc Surg. 2009; 37(3):262-71.

13. Vranic $H$, Hadzimehmedagic $A$, Haxibeqiri-Karabdic I, Mujacic E, Djedovic M. Critical Carotid Artery Stenosis in Coronary and Non-Coronary Patients - Frequency of Risk Factors. Med Arch (Sarajevo, Bosnia Herzegovina). $2017 ; 71(2): 110-4$.

14. Gates PC, Chambers B, Yan B, Chong W, Denton M. Symptomatic and asymptomatic carotid stenosis: just when we thought we had all the answers. Intern Med J. 2006; 36(7):445-51.
15. Roh Y-N, Woo S-Y, Kim N, Kim S, Kim Y-W, Kim D-I. Prevalence of asymptomatic carotid stenosis in Korea based on health screening population. J Korean Med Sci. 2011; 26(9):1173-7.

16. Karunanidhi K, Nanthini D, Sundaram V, Singaram $M$. A Study on prevalence of carotid artery stenosis in acute ischaemic stroke patients. J Evol Med Dent Sci. 2016; 5 (103).

17. de Weerd M, Greving JP, de Jong AWF, Buskens E, Bots ML. Prevalence of Asymptomatic Carotid Artery Stenosis According to Age and Sex: Systematic Review and Metaregression Analysis. Stroke. 2009;40(4):1105-13.

18. Chiquete E, Torres-Octavo B, Cano-Nigenda V, ValleRojas $D$, Dominguez-Moreno $R$, Tolosa-Tort $P$, et al. Caracterizacion de factores asociados con estenosis carotidea en una poblacion de alto riesgo. Rev Neurol. 2014;58(12):541-7.

19. Rodgers A, MacMahon S, Gamble G, Slattery J, Sandercock P WC. Blood pressure and stroke: an overview of published reviews. United Kingdom Transient Ischaem Attack Colab Gr. 1996;147-312.

20. Lawes CM, Bennett DA, Feigin VL RA. Blood pressure and stroke: an overview of published reviews. Stroke. 2004;35:1024.

21. Osvaldo Fustinoni. Consenso de estenosis carotídea.

22. Rodgers A, MacMahon S, Gamble G, Slattery J, Sandercock P, Warlow C. Blood pressure and risk of stroke in patients with cerebrovascular disease. The United Kingdom Transient Ischaemic Attack Collaborative Group. BMJ. 1996;

23. De Angelis $M$, Scrucca $L$, Leandri $M$, Mincigrucci $S$, Bistoni $S$, Bovi $M$, et al. Prevalence of carotid stenosis in type 2 diabetic patients asymptomatic for cerebrovascular disease. Diabetes Nutr Metab. 2003 Feb;16(1):48-55.

24. Ji R, Pan $Y$, Yan H, Zhang R, Liu G, Wang $P$, et al. Current smoking is associated with extracranial carotid atherosclerotic stenosis but not with intracranial large artery disease. BMC Neurol. 2017 Dec 26;17(1):120. 